\title{
Review
}

\section{How to Recruit the Correct RNA Polymerase? Lessons from snRNA Genes}

\author{
Oleksandr Dergai ${ }^{1}$ and Nouria Hernandez $\mathbb{D}^{1, \star}$
}

Nuclear eukaryotic genomes are transcribed by three related RNA polymerases (Pol), which transcribe distinct gene sets. Specific Pol recruitment is achieved through selective core promoter recognition by basal transcription factors (TFs). Transcription by an inappropriate Pol appears to be rare and to generate mostly unstable products. A collection of short noncoding RNA genes [for example, small nuclear RNA (snRNA) or 7SK RNA genes], which play essential roles in processes such as maturation of RNA molecules or control of Pol II transcription elongation, possess highly similar core promoters, and yet are transcribed for some by Pol II and for others by Pol III as a result of small promoter differences. Here we discuss the mechanisms of selective Pol recruitment to such promoters.

\section{Pol II and Pol III Transcribe Different Sets of Genes}

Pol II and Pol III transcribe strikingly different shares of the genome: about 20000 genes for Pol II versus about 700 genes for Pol III in the human genome (see GENCODE database). Pol II transcribes mRNA-encoding genes, long noncoding RNA genes, and some of the small nuclear RNA (snRNA) genes. Whereas Pol II transcription of long noncoding RNAs, which has come under renewed scrutiny in recent years, remains relatively unexplored, transcription of mRNA and snRNA genes has been studied for several decades. Genes from these last two classes differ in promoter and gene body structure (snRNA genes do not contain introns), and by the signals that direct $3^{\prime}$ end formation of the RNA: a polyadenylation signal (most mRNA-encoding genes) or an RNA stem-loop (SL) encoding structure (most histone genes) in mRNA-encoding genes versus a $3^{\prime}$ box in snRNA genes [1-8]. Pol III transcribes short, noncoding RNA genes encoding $5 S$ rRNA, tRNAs, RNAs involved in processes as varied as the control of Pol Il transcription elongation or the processing of other RNA molecules, and some short interspersed elements (SINEs) of unknown function [9].

Pol II and III genes differ in promoter structure and termination signals. For example, Pol III terminates transcription at simple signals consisting mainly of a run of $T$ residues $[9,10]$, a feature that does not stop Pol II and is, indeed, often present within Pol II genes. Moreover, maturation of Pol II and Pol III products involves different processing steps and machineries [11,12]. Transcription of a Pol II gene by Pol III or vice versa is therefore expected to produce mostly nonfunctional and unstable RNAs due to improper termination and processing, although some of these molecules may be processed into short regulatory RNAs, such as microRNAs. Selective Pol recruitment is thus likely to contribute to cell energetic economy.

\section{Pol II and Pol III Promoters}

The motifs found in Pol II core promoters (see Glossary) of metazoan mRNA transcription units are depicted in (Figure 1A, Key Figure). The most widespread are the initiator (Inr) (about 45\% of promoters) [13,14] and the TATA box (less than $25 \%$ of promoters) [15]. Figure $1 \mathrm{~B}$ shows the Pol II core promoters of mammalian snRNA genes, which are more homogenous and contain a single main core element, the proximal sequence element (PSE). The PSE is coupled to a

\section{Highlights}

With a few exceptions, Pol II and Pol III transcribe distinct sets of genes.

Pol II and Pol III promoters are generally very different in structure. The Pol II and III snRNA promoters are the exception; they are very similar to each other, a feature conserved in several species. As a result, some of these genes, notably the $\mathrm{RPPH} 1$ promoter, can recruit Pol II or Pol III in vivo.

The TATA box and PSE-type sequences (PSE in mammals and sea urchin, PSEA in Drosophila melanogaster, and USE in Arabidopsis thaliana) are the only well-defined core promoter elements that are shared by some Pol II and III promoters.

Selective Pol II or III recruitment by snRNA promoters is specified by promoter features that shift the equilibrium of competitive interactions towards recruitment of TFIIB or BRF2, and Pol II or III transcription machineries.

${ }^{1}$ Center for Integrative Genomics, Faculty of Biology and Medicine, University of Lausanne, Lausanne, Switzerland

\section{*Correspondence:} nouria.hernandez@unil.ch (N. Hernandez). 
regulatory element, the 'distal sequence element' (DSE), which binds factors that help stabilize the basal transcription machinery on the PSE $[3,16]$.

Figure 1C-E shows the three types of Pol III core promoters. Type 1 promoters are present in the $5 S$ genes, type 2 in most Pol III genes (i.e., tRNA genes and a few other genes), and type 3 in the spliceosomal U6 snRNA genes and another 15 or so genes, including those encoding RMRP and RPPH1 RNAs, required for maturation of rRNAs and tRNAs, respectively [9,17-20], 7SK RNA, involved in regulation of the Pol II transcription elongation factor PTEF-b $[21,22]$, the vault RNA component of vault particles, implicated in intracellular transport, drug resistance, and more recently regulation of autophagy [23-25], and the Y RNAs, implicated in DNA replication [26]. Type 1 and 2 Pol III promoters are largely located within the RNA coding sequence (Figure 1E) [one exception being a TATA box present upstream of the transcription start site (TSS) in the Schizosaccharomyces pombe type 2 promoters [27], whereas type 3 Pol III promoters reside upstream of the TSS $[28,29]$. Type 3 Pol III promoters are, moreover, strikingly similar to the Pol II snRNA promoters, containing a DSE and a PSE [30] with, in addition, a TATA box located downstream of the PSE, centered about 28 bp upstream of the TSS [31,32] (Figure 1B,C).

Pol II and Pol III Preinitiation Complexes

Most Pol II and Pol III core promoter elements are distinct, with the exception of the TATA box and the PSE, which can be found in both classes of promoters (Figure 1). Correspondingly, although Pol II and Pol III preinitiation complexes (PICs) are similar in topology and architecture, only two basal TFs can be found in both, the TATA box-binding protein (TBP) and the snRNA activating protein complex \{SNAPC; also known as PSE transcription factor (PTF) [33]\}, which binds to the PSE (Figure 1B,C) [34-36]. These factors perform, however, fundamentally different functions, with TBP playing a general role in transcription and SNAPC serving to recruit TBP and other factors to PSE-containing promoters.

TBP is involved in transcription by Pol I, II, and III as part of, respectively, the selectivity factor 1 (SL1), TFIID, and TFIIIB complexes, which contain different TBP-associated factors (TAFs) $[37,38]$. Thus, although TBP was discovered as a TATA box-binding factor, it is part of PICs assembled at TATA-less promoters, which in mammals include Pol I, and the large majority of Pol II and Pol III promoters. At such promoters, TBP is recruited through protein-protein and protein-DNA interactions involving the TAFs $[39,40]$. For example, the DNA binding function of TBP is not required for transcription from TATA-less Pol II mRNA promoters [41], where TBP is recruited through interactions of different TFIID TAFs with the Inr, the downstream promoter element (DPE), or the motif ten element (MTE; Figure 1; see $[42,43]$ and references therein).

If most promoters are TATA-less, why is TBP so generally present in PICs? The answer may be in its ability to recruit, through protein-protein contacts, the members of the TFIIB family of TFs (Box 1), namely TAF1B, TFIIB, or for Pol III the TFIIB-related factors BRF1 or BRF2 as well as another factor, BDP1. These proteins associate directly not only with TBP but also, highly selectively, with Pol I, Pol II, or Pol III [37,44-46]. The TBP-mediated incorporation of TAF1B, TFIIB, or one of the BRFs together with BDP1 within the assembling PIC is thus a decisive step in the recruitment and selection of the correct Pol. The role of basal DNA binding factors such as TFIIA, which stabilizes TBP and TFIIB on Pol II promoters (Figure 1A,B) $[40,47]$, or TFIIIA and/or TFIIIC, which form the platform allowing recruitment of TBP together with BRF1 and BDP1 on type 1 and 2 Pol III promoters (Figure 1D,E) $[35,48]$, is thus largely to favor the recruitment of TBP associated with the correct TFIIB family member. Consistent with TFIIIA and TFIIIC being dispensable for Pol III transcription, a combination of a TATA box and TBP, BRF1, and BDP1 is sufficient to direct Pol III transcription in vitro [49,50]. Further, the human C-Myc promoter and various TATA box containing core Pol II promoters can drive both Pol II and Pol III
Glossary

Core promoter: the promoter region capable of recruiting, in vitro, the basal transcription machinery, comprising the basal TFs and the Pol. It is generally located within 100 base pairs upstream and downstream of the TSS.

Regulatory elements: DNA

sequences that can be located far away from the TSS and that contain binding sites for SSTF that activate or repress transcription from the core promoter. SSTF binding can be cooperative with the binding of basal TFs. 


\section{Key Figure}

Pol II and III Promoters and Components of the Corresponding PICs

\section{(A)}

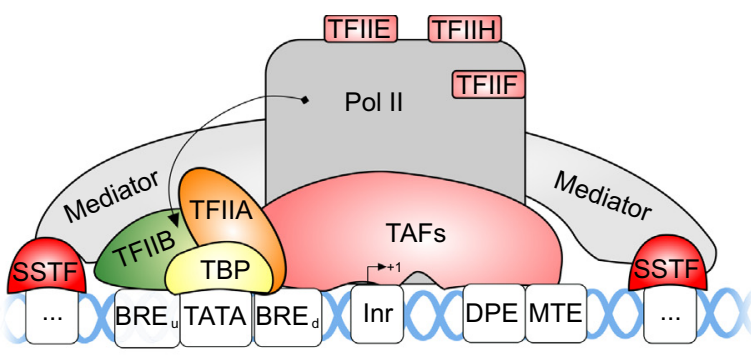

(B)

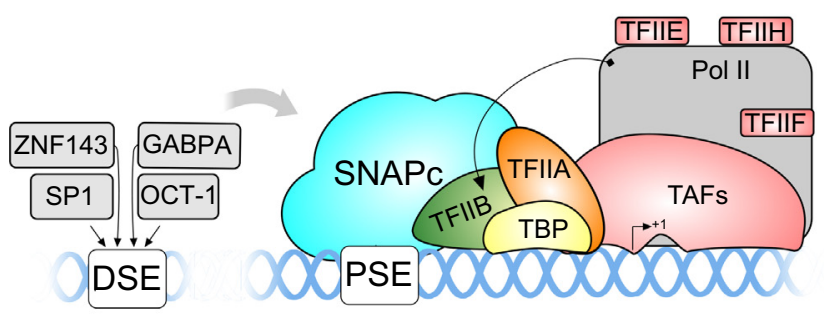

(C)

Type 3

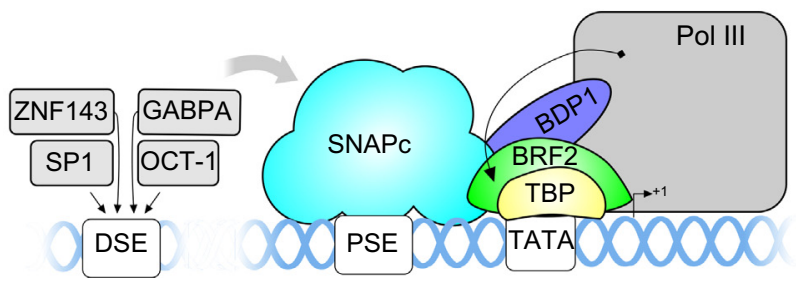

PolyA-signal

(D)

\section{Type 1}

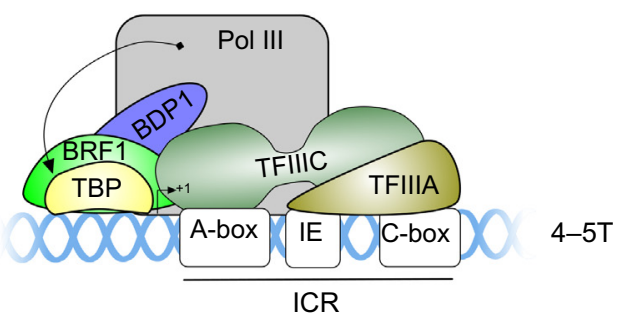

(E)

\section{Type 2}

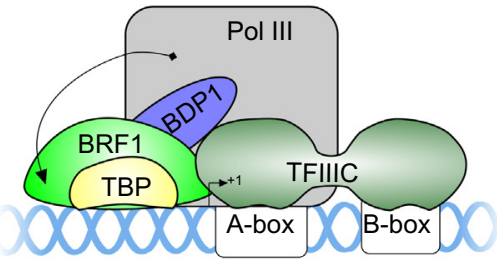

Figure 1. (A) Generic Pol II mRNA promoter. Different core promoters contain different combinations of the depicted motifs. Transcription factor IID (TFIID), composed of TATA box-binding protein (TBP) and TBP-associated factors (TAFs), is recruited through TBP-TATA box interactions and/or TAFs interactions with the initiator (Inr), the downstream promoter element (DPE), or the motif ten element (MTE). In certain promoters, TFIIB recognition elements located either upstream (BRE ${ }_{\mathrm{u}}$ ) or downstream (BRE $E_{d}$ ) of the TATA box help recruit TFIIB. TFIIA binds to and stabilizes the TBP-DNA complex. TFIIB interacts directly with Pol II. Various regulatory elements recruit site-specific transcription factors (SSTF) that can act on the preinitiation complex (PIC) through contacts with the general transcription machinery or with subunits of the mediator, a large complex with both activating and repressing modules (see [97] for a review). TFIIF, TFIIE, and TFIIH are required for transcription initiation (see [43] for a review). (B) Pol II small nuclear RNA (snRNA) promoter. The core promoter consists of the proximal sequence element (PSE), which recruits the snRNA activating protein complex (SNAPC). The PIC contains, in addition, TBP together with some of the TFIID TAFs, TFIIA, and TFIIB, which recruits Pol II, TFIIF, TFIIE, and perhaps TFIIH $[54,98,99]$. Factors binding to the distal sequence element (DSE) activate transcription, at least in part, by stabilizing the PIC on the core promoter. (C) Type 3 Pol III promoter. The promoter contains a PSE and DSE interchangeable with the PSE and DSE of Pol II snRNA promoters and, in addition, a TATA box. The PIC contains 
Box 1. The TFIIB Family of Transcription Factors

All known multisubunit cellular RNA Pols require accessory protein factors for specific recruitment to their cognate promoters, from bacterial Pol, which uses $\sigma$ factors, to archaeal and eukaryotic Pols, which require several factors, among them members of the TFIIB family of transcription factors. Remarkably, bacterial $\sigma$ factors, archaeal TFB, and eukaryotic TFIIBs are in fact structural homologs and share the property of binding directly to the Pol with part of the protein very close to the catalytic core [101]. However, whereas the $\sigma$ factor binds directly to promoter sequences, efficient binding of TFIIB family members to promoter core sequences requires the help of other proteins such as TBP and/or TBP-associated factors (TAFs), and TFIIB can be seen as bridging DNA-bound TBP/TAFs with Pol II. Thus, although TFIIB, for example, recognizes the $\mathrm{BRE}_{\mathrm{u}}$ and $\mathrm{BRE}_{\mathrm{d}}$ elements present upstream and downstream of the TATA box in some promoters, these interactions are not sufficient for recruitment in the absence of TBP. TFIIB family members retain, however, other properties of $\sigma$ factor, such as stimulation of RNA chain synthesis [102].

transcription in injected Xenopus oocytes and HeLa cell extracts, the choice of the Pol being determined by the ratio of template to factors [51-53]. This confirms that a TATA box can assemble both Pol II and Pol III PICs and suggests that specific Pol recruitment is determined by competitive binding of basal TFs to the template. As described later, SNAPc is an interesting assembly factor that unlike TFIIA, TFIIIA, or TFIIIC, can promote the recruitment of TBP and either BRF2 or TFIIB, depending on the presence or absence of a TATA box [54] (see Box 2).

In addition to the basal TFs TBP and SNAPc, the Pol II and III transcription machineries can share regulatory factors such as Oct-1, Sp1, GABPA, or ZNF143 ([55] and references therein), which can bind to the DSEs of Pol II and III PSE-containing promoters as well as to mRNA Pol II promoters, or Myc [56], Rb [57,58], NF-kB [59], and p53 [60], which are recruited to Pol II and III promoters through interactions with DNA elements or the basal transcription machinery. These factors contribute to transcription regulation, but not to the selectivity of Pol recruitment.

\section{PSE-Containing Promoters: Structure and Features in Different Species}

Curiously, although the promoter structures themselves vary in different species, the similitude of snRNA Pol II and III promoters has been conserved in several species of animals and plants. Indeed, the only known example so far of a gene transcribed by different Pol in different eukaryotes is the U3 snRNA gene. This gene is transcribed by Pol III in monocots and dicots, but by Pol II in vertebrates and lower eukaryotes including yeast [61], suggesting a change from Pol II to Pol III specificity in an angiosperm ancestor of monocots and dicots [62].

The first demonstration that the specificity of Pol II and III snRNA promoters could be easily altered was provided with manipulations of the Xenopus tropicalis U6 snRNA gene [28]. Precise mutagenesis in the mammalian genes then showed that a TATA box, located between the PSE and the TSS, is a dominant element triggering Pol III over Pol II specificity, whereas the PSEs contribute little to Pol specificity (Figure 2) [31]. The position of the TSS is defined largely by the PSE for Pol II, and the TATA box for Pol III genes [31].

In Drosophila melanogaster, the core promoter of snRNA genes consists of a proximal sequence element A (PSEA) sequence, slightly different in the Pol II and Pol III promoters, followed by a proximal sequence element B (PSEB) sequence in the Pol II, and by a TATA box in the Pol III promoters (Figure 2) $[63,64]$. The key determinant of Pol specificity is not presence or absence of the TATA box, but rather the precise sequence of the PSEA element. The spacing between the PSEA and PSEB, or the PSEA and the TATA box, is crucial for efficient transcription but does not contribute

SNAPc bound to the PSE, TBP bound to the TATA box, as well as the TFIIB-related factor 2 (BRF2) and another factor BDP1, which recruit Pol III. TBP, BRF2, and BDP1 form the BRF2-TFIIIB complex. (D) Type 1 Pol III promoter. The promoter is gene-internal and consists of an internal control region (ICR) composed of A and C boxes separated by an intermediate element (IE). The ICR recruits the zinc finger protein TFIIIA, followed by the TFIIIC complex. The BRF1-TFIIIB complex, composed of TBP, the TFIIB-related factor 1 (BRF1), and BDP1, can then join the PIC and recruit Pol III. (E) Type 2 Pol III promoter. The promoter is gene-internal and consists of A and B boxes, which directly recruit TFIIIC. The PIC then assembles like on type 1 Pol III promoters. 


\section{Box 2. SNAPc in Different Species}

The specific features of snRNA promoters that dictate which of either Pol II or Pol III is recruited are different in different species. Yet, at least in the species where it has been characterized, namely human and mouse, Drosophila melanogaster, and trypanosomes, the PSE-binding factor turns out to be conserved (see [103] for a review). Mammalian SNAPc is composed of five subunits: SNAP190, SNAP50, SNAP45, SNAP43, and SNAP19; SNAP190 constitutes the backbone of the complex and contains a four-and-a-half Myb repeat DNA-binding module [36]. A subcomplex consisting of a truncated SNAP190, SNAP19, SNAP43, and SNAP50, referred to as mini-SNAPC, is competent for PSE binding and in vitro transcription $[16,104]$. Interestingly, Drosophila SNAPc is very similar to mammalian mini-SNAPc, with a large subunit corresponding to the truncated SNAP190 of mini-SNAPc and smaller subunits resembling SNAP43 (DmSNAP43) and SNAP50 (DmSNAP50) [91].

In trypanosomes, there is only one snRNA synthesized by Pol II, the so-called SL RNA, a short RNA involved in the processing of polycistronic RNAs into individual mRNAs by trans-splicing $[105,106]$. SL RNA transcription requires trypanosome SNAPc (tSNAPc), whose three subunits clearly resemble the core SNAP190, SNAP50, and SNAP43 human SNAPc subunits $[103,107]$. tSNAPc does not appear, however, to be required for transcription of any trypanosome Pol III genes [108].

to the selectivity of Pol recruitment. In this case the position of the TSS is defined by the PSEA for both Pol II and Pol III genes [63,65].

In Arabidopsis thaliana, Pol II and Pol III transcription of snRNA genes requires the same two basal promoter elements: an 'upstream sequence element' (USE) and a TATA box [66]. In maize, an additional element, monocot-specific promoter element (MSP), located upstream of the USE, is required for efficient transcription [67]. Pol specificity is determined by the spacing between the USE and TATA box: 32-36 bp spacing specifies Pol II transcription whereas 23-26 bp spacing specifies Pol III transcription (Figure 2) [61,68]. A small deletion between these two elements may thus be at the origin of the change in Pol specificity in plant U3 snRNA genes.

snRNA promoters have been examined in other species, but in these cases the elements determining specific Pol II recruitment have not been identified. Thus, in zebrafish, some of the U6 snRNA promoters contain a putative DSE with a CCAAT sequence as well as clearly recognizable PSE and TATA box [69]. In chicken, the Pol II U1, U2, U4, and Pol III U6 snRNA genes contain virtually identical PSEs and DSEs [70-72]. In sea urchin, snRNA genes have functional DSE and PSE equivalents that differ in different snRNA genes and have no similarity to the mammalian elements [73-75]. The PSEs in the sea urchin Pol II and Pol III genes are interchangeable and form similar DNA-protein complexes as determined by electrophoretic mobility shift assays (EMSAs), suggesting that like the PSE in other species (see later), they bind the same factors [76]. Curiously, both the Pol II U2 and the Pol III U6 snRNA promoters have, in addition to the PSE, an essential TATA box (Figure 2) [73], a feature not seen in Pol II snRNA promoters of other animal species.

\section{Molecular Mechanisms of Selective Pol Recruitment to PSE-Containing Promoters}

Current knowledge of PIC assembly at snRNA promoters results mainly from in vitro studies of partial PIC reconstitution, in vitro transcription assays, and genome-wide studies of specific TFs and Pol localization. Selective Pol recruitment to PSE-dependent genes is best understood in mammals and Drosophila. A key step in both systems is recognition of the PSE by SNAPC Box 2. At human promoters, where the PSE is coupled to an upstream DSE, often containing an OCT-1 binding site, OCT-1 and SNAPc bind cooperatively to their respective binding sites thanks to a protein-protein contact between the two factors that relieves autoinhibition of SNAPc DNA binding [77]. The assembly of a Pol II or Pol III specific PIC is, however, determined by specific features of the core promoter.

Pol II PICs appear to assemble differently at PSE-containing (and TATA-less) promoters than at mRNA promoters. In the latter case, assembly of the PIC involves the recruitment of the TFIID 


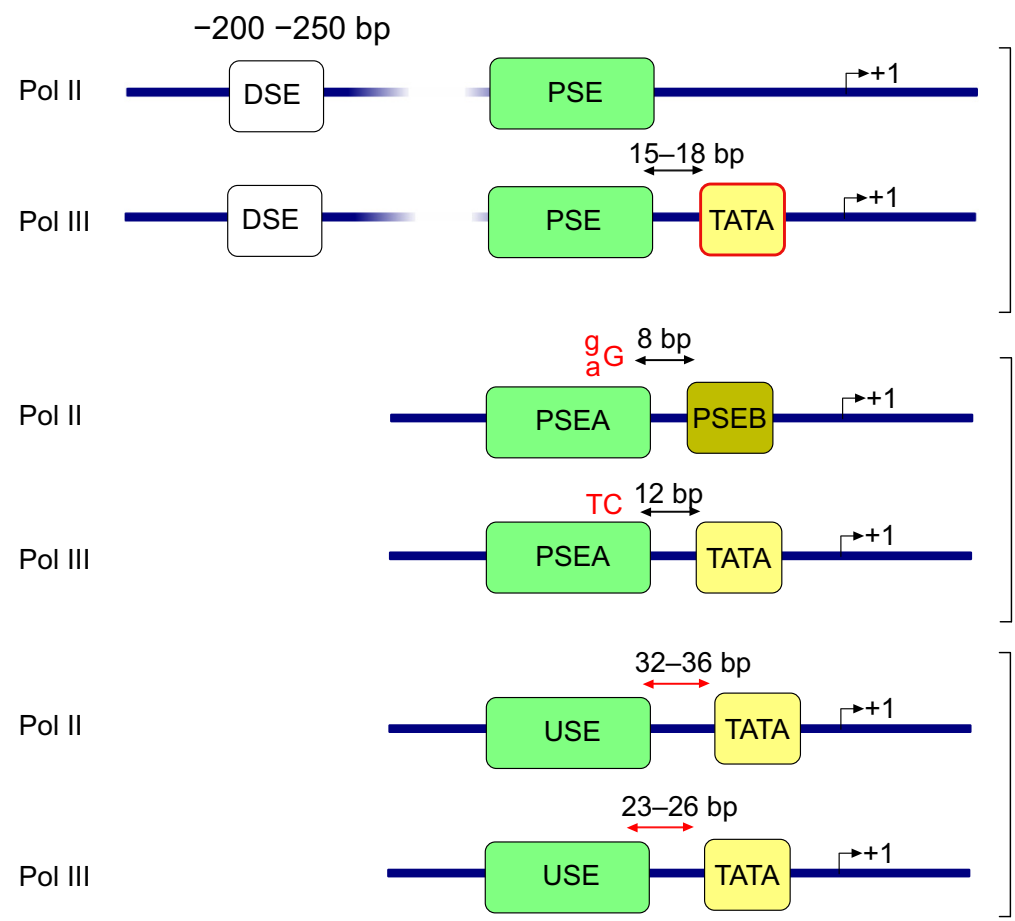

Human

Fly

Arabidopsis

Pol II U1
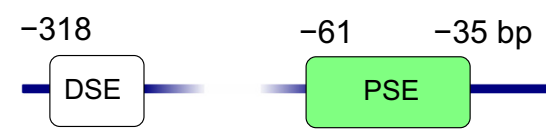

Pol II (U2)

Pol III (U6)
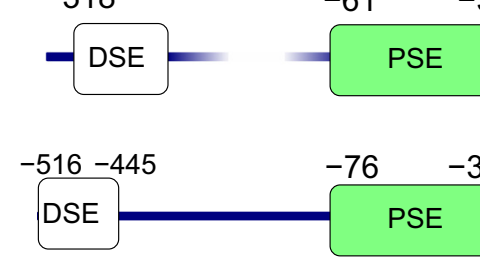

\section{$15 \mathrm{bp}$}

$-76 \quad-38$

PSE

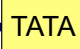

Sea urchin

Trends in Genetics

Figure 2. Structure of Human, Drosophila, Arabidopsis, and Sea Urchin PSE-Containing Promoters. The elements that dictate Pol specificity are indicated in red. The determinants of Pol specificity in the sea urchin promoters are not known. Abbreviations: DSE, distal sequence element; E-box, enhancer box; PSE, proximal sequence element; PSEB, proximal sequence element B; USE, upstream sequence element.

complex, which binds to the TATA box and/or the Inr element through contacts with TBP and with TAFs, respectively (Figure 1A; [43] and references therein). TFIIA allows efficient TBP-DNA interaction by displacing the $\mathrm{N}$-terminal portion of TAF1 from the concave DNA-binding surface of TBP, and by stabilizing the TBP-TATA box complex [40]. TFIIB is recruited via a direct interaction with TBP involving the C-terminal stirrup of TBP and the TFIIB core domain [78], an event that then allows the recruitment of Pol II [45,79]. Thus, in mRNA promoters, the TATA box cooperates with other core promoter motifs to recruit the TBP-containing complex TFIID $[15,43]$.

At PSE-containing promoters, TFIIB is recruited by protein-protein contacts with SNAPC. BRF2 can also bind directly to SNAPC, but the binding of TFIIB and BRF2 is mutually exclusive, 

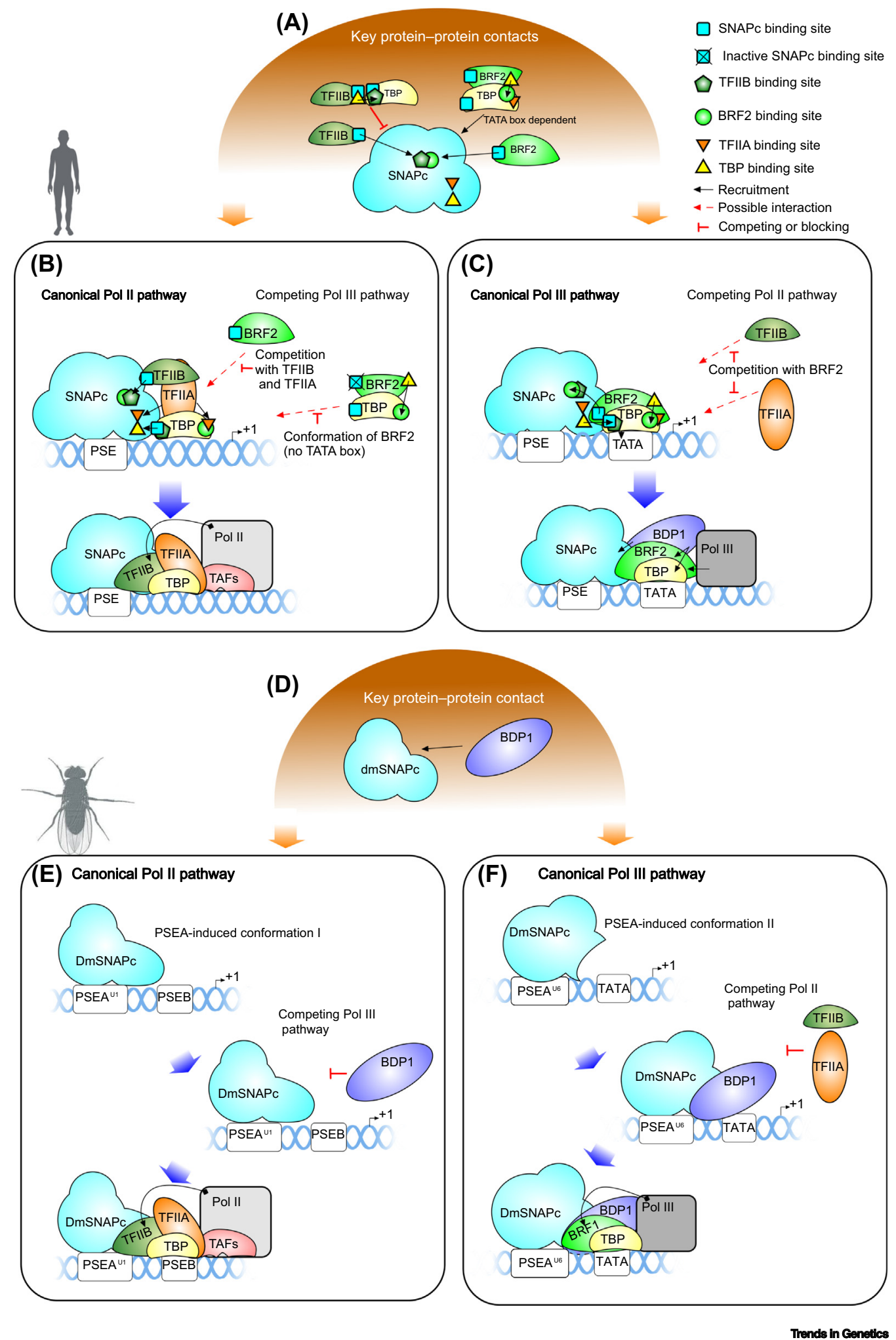

(See figure legend at the bottom of the next page.) 
suggesting that these two proteins interact with overlapping SNAPC surfaces (Figure 3A, olive green pentagon and light green circle on SNAPC). Remarkably, however, the binding of TFIIB to SNAPC is strongly decreased if TFIIB is first complexed with TBP. Indeed, in this case, (i) the TFIIB surfaces used to bind to SNAPC and to TBP (Figure 3A, turquoise square and yellow triangle on TFIIB) overlap, and (ii) the TBP surfaces used to interact with SNAPC and with TFIIB (Figure 3A, olive green pentagon and turquoise square on TBP) overlap. This latter observation strongly suggests that on Pol II PSE-containing promoters, TBP and TFIIB are recruited separately from each other (Figure 3B, upper drawing), unlike at TATA box containing mRNA promoters. Nevertheless, it seems likely that after separate recruitment of TBP and TFIIB, the proteins rearrange inside the maturing complex to reach a final conformation close to that achieved at Pol II mRNA promoters (Figure 3B, lower drawing) [54].

TFIIA can directly contact SNAPC (Figure 3A,B, upper drawing, orange triangle on SNAPC), which strengthens the SNAPC-TBP interaction [54]. Importantly, TFIIA contributes to the specific assembly of a Pol II PIC at PSE-containing TATA-less promoters by competing with BRF2, but not with TFIIB, for binding to TBP, consistent with two overlapping surfaces of TBP binding to BRF2 and TFIIA (Figure 3A,B, upper drawing, light green circle and orange triangle on TBP); [80]. Thus at TATA-less promoters, both TFIIB and TFIIA, the two canonical Pol II TFs, contribute to shifting the equilibrium towards Pol II PIC assembly [54].

Another effect possibly contributing to favoring Pol II recruitment at TATA-less promoters is a property of TFIIB. TFIIB exists in two conformations [81-83]: in its 'closed' conformation, a charged cluster domain (CCD) located in its $\mathrm{N}$-terminal region occludes its core domain and thus prevents efficient SNAPc binding; in its 'open' conformation, the intramolecular binding of the CCD to the core domain is disrupted. A truncated TFIIB lacking the CCD has much higher affinity for SNAPC than full-length TFIIB, suggesting that the same would be true for TFIIB in an open conformation [54]. Such a large difference in affinity is not observed in a comparison of full-length BRF2 and BRF2 core domain, and importantly, truncated TFIIB has higher affinity for SNAPc than BRF2 [54]. Thus, in the presence of transcriptional activators bound to the DSE and perhaps causing TFIIB to adopt an open conformation, TFIIB would have a competitive advantage over BRF2 in binding to SNAPc when the PSE is the sole core promoter element [54].

At mammalian PSE and TATA box-containing promoters, Pol III specificity is triggered by simultaneous recognition of the PSE by SNAPC and the TATA box by TBP (Figure 3C). The distance separating the PSE and the TATA box is relatively constant in different promoters, and an increase

Figure 3. Assembly of Pol II or III PICs at PSE-Containing Promoters. (A) Key interactions (black arrows) that trigger selective Pol II or III preinitiation complex (PIC) assembly in mammals. Transcription factor IIB (TFIIB) and TFIIB-related factor 2 (BRF2) can both bind to small nuclear RNA activating protein complex (SNAPC), but in a mutually exclusive manner, suggesting they bind overlapping SNAPc surfaces. A TFIIB-TBP complex cannot interact with SNAPc (red line), because of overlaps between: (i) the TATA box-binding protein (TBP) and SNAPc binding surfaces in TFIIB, and (ii) the TFIIB and SNAPc binding surfaces in TBP. A BRF2-TBP complex can interact with SNAPc bound to the proximal sequence element (PSE) only upon binding to a TATA box located at the correct distance downstream of the PSE. In this complex, the TFIIA binding site on TBP is occluded by BRF2. (B) Assembly of a mammalian Pol II PIC. Upper drawing: TBP and TFIIB are recruited separately to the SNAPc-PSE complex via their SNAPc-interacting surfaces. TFIIA can bind to SNAPc and to TBP, whose TFIIA binding site is not occluded. BRF2 cannot join the complex because its binding site on SNAPc is occluded by TFIIB and its binding site on TBP is occluded by TFIIA. As there is no TATA box, the SNAPc binding site of BRF2 in the BRF2-TBP complex is not available for binding to SNAPc, probably because of inadequate conformation. Lower drawing: the complex may rearrange to acquire a TBP-TFIIB-TFIIA conformation close to that in mRNA promoters. (C) Assembly of a mammalian Pol III PIC. Upper drawing: in the presence of both a PSE and a TATA box, the TBP-BRF2 complex acquires a conformation such that the SNAPc binding site on BRF2 is available, and thus TBP and BRF2 both contact SNAPc. BRF2 occludes both the TFIIB binding site on SNAPC and the TFIIA binding site on TBP, preventing these proteins from joining the complex. Lower drawing: the incorporation of BDP1 into the complex allows efficient transcription initiation by Pol III. In panels B and C, dashed red arrows indicate competing interactions and red solid lines indicate inhibitory mechanisms. (D) Key interaction that triggers selective Pol II or III PIC assembly in Drosophila melanogaster. BDP1 directly binds to SNAPc, and this interaction is conditioned by a SNAPc conformation dictated by the specific proximal sequence element A (PSEA) sequence. (E) Assembly of a D. melanogaster Pol II PIC. The PSEA sequence in the Pol II U1 snRNA promoter (PSEA ${ }^{\mathrm{U1}}$ ) causes DmSNAPc to adopt a conformation in which the BDP1 binding site is not available, allowing Pol II PIC assembly. (F) Assembly of a D. melanogaster Pol III PIC. On the PSEA sequence of the Pol III U6 snRNA promoter (PSEA ${ }^{U}$ ), DmSNAPc adopts a conformation which allows BDP1 binding, presumably creating a platform for BRF1 and TBP recruitment $[91,92,100]$. This in turn shifts the equilibrium to Pol III PIC assembly. 
of 3-4 bp or more strongly diminishes SNAPc-TBP complex formation and transcription [34], consistent with the observed cooperative binding of SNAPC and TBP to the U6 promoter [84]. BRF2 recruitment is then favored by several cooperative interactions (Figure 3C, upper drawing): with SNAPC, TBP, and DNA. BDP1 further stabilizes the PIC by binding to the BRF2-TBP heterodimer, to SNAPC, and to DNA just upstream of the TATA box (Figure 3C, lower drawing) [85]. Thus, efficient BDP1 recruitment occurs after Pol specificity has been determined by recruitment of BRF2, and in this sense does not play a role in determination of Pol specificity [54].

The most important factor for favoring Pol III recruitment at TATA-containing promoters is probably TBP. Indeed, in vitro, in the absence of TBP, the SNAPC-BRF2 binary complex is not specific with regard to promoter sequence, it can be assembled at any PSE containing promoter, whether a TATA box is present or not (Figure 3A) [54]. Specificity arises upon addition of TBP, which enhances BRF2 association at TATA box containing promoters (Figure 3C), and most importantly, prevents BRF2-SNAPc interaction in the TATA-less context (Figure 3B, upper drawing) [54]. This is consistent with the hypothesis that, in the context of a PSE and a downstream TATA box, SNAPC, TBP, and BRF2 form a complex in which BRF2 has a different conformation than when in a heterodimer with TBP off the DNA (Figure 3A,B, upper drawing). BRF2 requires a region referred to as 'the arch' to assemble with SNAPC and TBP on PSE-containing and TATAcontaining probes in EMSAs [80]. Perhaps the arch adopts the correct conformation only when BRF2 is in a complex with TBP bound to the TATA box downstream of the PSE (Figure 3B,C, upper drawings, turquoise square on BRF2).

Since BRF2, when not associated with TBP, can bind to DNA-bound SNAPc in vitro provided that SNAPC is not bound by TFIIB, and since BRF2 specifically associates with Pol III, one could imagine that a SNAPC-BRF2 complex might assemble on TATA-less snRNA promoters and erroneously recruit Pol III. However, only traces of BRF2, and no BDP1, were detected at such promoters in cultured cells by chromatin immunoprecipitations [54]. Thus, a BRF2SNAPc complex rarely assembles in vivo at TATA-less snRNA promoters and is probably transcriptionally inactive as it does not detectably recruit BDP1 [49,50,85].

In D. melanogaster (Dm), the PSEAs of Pol II and III promoters carry essential pol specificity information [63,86-88], and are thus not interchangeable, unlike the PSEs in the mammalian system. Careful crosslinking and EMSA studies have shown that DmSNAPc adopts alternative conformations dictated by the type of PSE it is associated with (Figure 3E,F) $[89,90]$. One of its subunits, DmSNAP43, contacts approximately 20 bp of DNA downstream of the Pol II U1 PSEA, but only about 5 bp downstream of the Pol III U6 PSEA [91]. The specific DmSNAPc conformation is sensed by BDP1, which associates efficiently with DmSNAPc bound to a U6 PSEA (Figure 3F) but not a U1 PSEA (Figure 3E) [92]. Conceivably, the BDP1 binding surface of DmSNAPc is occluded or inappropriately folded in the U1 PSEA-bound context. Formation of a PSEA-bound DmSNAPc-BDP1 complex presumably creates a platform for subsequent TBP, BRF1, and Pol III recruitment (Figure 3F). On Pol II promoters, the inability of BDP1 to bind DmSNAPc associated with a U1-type PSEA might leave DmSNAPc interfaces vacant for the binding of TFIIB and other Pol II-specific factors (Figure 3E).

The mechanisms of selective Pol recruitment operating in mammals and fly appear different, with the sequence-induced different DmSNAPc conformations being critical in the fly. However, although the mammalian Pol II and Pol III PSEs are interchangeable, chromatin immunoprecipitation experiments with antibodies directed against different SNAPc subunits reveal striking shifts in tag densities, with two SNAPc subunits displaying peaks closer to the TSS in Pol III than in Pol II promoters [55]. Thus, like in flies, SNAPC adopts different conformations on Pol II and III promoters, but the different conformations most likely result from protein-protein contacts with 
TATA-bound TBP and BRF2 in the Pol III promoters rather than from the PSE sequence per se. The same general principle may apply in plants, where the key determinant of Pol specificity is spacing between the USE and the TATA box. Here also, the SNAPC conformation may be dictated by different protein-protein contacts with TATA-bound TBP imposed by the location of the TATA box relative to the USE. The key principles leading to specific Pol II or III recruitment on mammalian PSE-containing promoters are stepwise recruitment, rather than recruitment as a complex, of TBP and TFIIB on Pol II PSE-containing promoters, and highly favored interaction of a BRF2-TBP complex over a TFIIB-TBP complex with PSE-bound SNAPC in the context of a TATA box containing promoter. Perhaps, then, regardless of promoter differences in different species, these key principles also hold for PSE-containing promoters in Drosophila and other species.

\section{Dual Pol II and Pol III Transcription}

The high similarity of Pol II and Pol III PSE-containing promoters raises the possibility of their widespread dual occupancy in vivo. Yet, although the U6, 7SK, and RPPH1 promoters can direct luciferase or CRISPR-Cas9 (clustered regularly interspaced palindromic repeats-CRISPR associated protein 9) expression by Pol II transcription from transfected plasmids [93,94], only the RPPH1 promoter is convincingly occupied by Pol II and Pol III within the genomic context [55]. This promoter displays the longest distance separating the PSE and the TATA box. Decreased Pol recruitment selectivity could thus result from weaker cooperative binding of SNAPC and TBP to their respective targets, which in turn would allow occupancy by either SNAPc alone bound to the PSE, leading to recruitment of TFIIB as described earlier (Figures 1B and 3B), or to a TBP-TFIIB complex bound to the TATA box leading to a mRNA type Pol II transcription initiation complex (Figure 1A). The first possibility seems more likely, as transfected RPPH1 promoter-luciferase constructs direct a Pol II TSS 8 bp upstream of the Pol III TSS [93], that is, at a distance downstream of the PSE typically observed at Pol II snRNA promoters and at a U6 promoter with a mutated TATA box [31]. Indeed, efficient expression of protein coding genes transcribed from the mouse Pol II U1 snRNA promoter has been observed in transfected cells, an observation consistent with the known capping mechanism of Pol II snRNAs [95], which first acquire the conventional 7-monomethylguanosine cap recognized by the translational machinery. The further methylation leading to a trimethyl $G$ cap occurs after association with small nuclear ribonucleoproteins (snRNPs), a step that will not occur for an RNA lacking a snRNA sequence [96].

\section{Concluding Remarks}

Pol II and III promoters are widely different in structure except for the Pol II snRNA promoters and the Pol III type 3 promoters, which share common promoter elements and whose Pol specificity can be easily altered with small promoter sequence changes. The resemblance of these Pol II and III promoters is conserved in different species, even when the promoter elements themselves are not, and so too is the use of a common and specialized SNAPc-type TF. Understanding whether this conserved resemblance of Pol II and Pol III snRNA promoters affords a biological advantage will be a challenge. A possibility is a need for coregulation, which seems natural in the case of certain snRNA genes whose products function together, for example, in pre-mRNA splicing, but whose role in other cases will be difficult to establish. Indeed, RNAs transcribed from PSEcontaining promoters function at numerous steps of gene expression, whose links are not fully understood.

PSE-containing Pol III promoters have several properties that make them highly suitable for in vivo expression of short RNAs, and indeed they are commonly used for such purpose. They are strong promoters, they are located upstream of the transcribed region and thus do not constrain the sequence of the RNA, and they fire a polymerase that terminates transcription at simple runs
Outstanding Questions

What are the 3D structures of SNAPc in Pol II and Pol III PICs?

What are the 3D structures of DmSNAPC bound to a PSEA ${ }^{\mathrm{U} 1}$ and DmSNAPC bound PSEA ${ }^{\mathrm{U}}$ ? Are the structural changes similar to those observed with mammalian SNAPc in Pol II and Pol III PICs?

What are the molecular mechanisms leading to specific Pol II or III recruitment in different species such as sea urchin or Arabidopsis thaliana? Do they suggest some general principles applicable to all snRNA-type genes?

Are the positions of TBP and TFIIB in the final PoI II PIC assembled on a PSE different from their positions in a Pol II PIC assembled on a TATA box?

Are the 'open' and 'closed' conformations of TFIIB playing a role in Pol II snRNA gene transcription?

Are the Pol II and Pol III PSE-containing promoters coregulated? Under which circumstances?

Is transcription of the RPPH1 gene, and perhaps other genes with PSEcontaining promoters, by both Pol II and Pol III of any functional significance?

Why are some snRNAs transcribed by Pol II rather than Pol III, like most short RNAs? Is it because Pol III would often recognize the internal Sm binding site, required for assembly of the RNA with Sm proteins to form an snRNP, as a termination signal, due to it containing a short run of $\mathrm{T}$ residues? 
of T residues. Even if dual transcription of PSE-containing promoters by Pol II and Pol III appears to be the exception rather than the rule, it seems important when such promoters are used for therapeutic purposes, to optimize not only transcription levels by Pol III, but also to ensure absence of transcription by Pol II, as such transcription might lead to longer RNAs containing potentially harmful sequences (see Outstanding Questions).

\section{Acknowledgments}

We thank Dr M.C. Gambetta and P. Cousin for critical comments on this review. We also thank the reviewers for their comments, which improved the manuscript. This work was funded by the University of Lausanne and grant 31003A_169233 from the Swiss National Science Foundation.

\section{References}

1. Hernandez, N. (1985) Formation of the $3^{\prime}$ end of U1 snRNA is directed by sequence located downstream of the coding region. EMBO J. 4, 1827-1837

2. Ach, R.A. and Weiner, A.M. (1987) The highly conserved $U$ small nuclear RNA $3^{\prime}$-end formation signal is quite tolerant to mutation. Mol. Cell. Biol. 7, 2070-2079

3. Hernandez, N. (2001) Small nuclear RNA genes: a model sys tem to study fundamental mechanisms of transcription. J. Biol. Chem. 276, 26733-26736

4. Egloff, S. et al. (2008) Expression of human snRNA genes from beginning to end. Biochem. Soc. Trans. 36, 590-594

5. Proudfoot, N.J. (2016) Transcriptional translation in mammals: stopping the RNA polymerase II juggernaut. Science 352, aad9926

6. Krieg, P.A. and Melton, D.A. (1999) Formation of the $3^{\prime}$ end of histone mRNA. Gene 239, 203-206

7. Marzluff, W.F. (1992) Histone $3^{\prime}$ ends: essential and regulatory functions. Gene Expr. 2, 93-97

8. Marcela, D.L. and Samuelsson, T. (2008) Early evolution of histone mRNA $3^{\prime}$ end processing. RNA 14, 1-10

9. Schramm, L. and Hernandez, N. (2002) Recruitment of RNA polymerase III to its target promoters. Genes Dev. 16, 2593-2620

10. Arimbasseri, A.G. and Maraia, R.J. (2016) Mechanism of transcription termination by RNA polymerase III utilizes a nontemplate-strand sequence-specific signal element. $\mathrm{Mol}$. Cell 58, 1124-1132

11. Bentley, D.L. (2014) Coupling mRNA processing with transcription in time and space. Nat. Rev Genet. 15, 163-175

12. Kufel, J. and Grzechnik, P. (2019) Small nucleolar RNAs tell a different tale. Trends Genet. 35, 104-117

13. Ngoc, L.V. et al. (2017) The human initiator is a distinct and abundant element that is precisely positioned in focused core promoters. Genes Dev. 31, 6-11

14. Piero, C. et al. (2006) Genome-wide analysis of mammalian promoter architecture and evolution. Nat. Genet. 38, 626-636

15. Kadonaga, J.T. (2012) Perspectives on the RNA polymerase II core promoter. Wiley Interdiscip. Rev. Dev. Biol. 1, 40-51

16. Mittal, V. et al. (1999) Snap : a core promoter factor with a built-in DNA-binding damper that is deactivated by the Oct- 1 POU domain. Genes Dev. 13, 1807-1821

17. Chamberlain, J.R et al. (1996) An RNase P RNA subunit mutation affects ribosomal RNA processing. Nucl. Recept. Signal. 24, 3158-3166

18. Goldfarb, K.C. and Cech, T.R. (2017) Targeted CRISPR disruption reveals a role for RNase MRP RNA in human preribosomal RNA processing. Genes Dev. 31, 59-71

19. Guerrier-Takada, C. et al. (1983) The RNA moiety of ribonuclease $\mathrm{P}$ is the catalytic subunit of the enzyme. Cell 35, 849-857

20. Bartkiewicz, M. et al. (1989) Identification and characterization of an RNA molecule that copurifies with RNase $\mathrm{P}$ activity from HeLa cells. Genes Dev. 3, 488-499

21. Peterlin, B.M. et al. (2012) 7SK snRNA: a noncoding RNA that plays a major role in regulating eukaryotic transcription. Wiley interdiscip. Rev. RNA 3, 92-103

22. Diribarne, G. and Bensaude, O. (2009) 7SK RNA, a noncoding RNA regulating P-TEFb, a general transcription factor. RNA Biol. 6, 122-128
23. Chen, J. et al. (2018) Vault RNAs partially induces drug resisance of human tumor cells MCF-7 by binding to the RNA DNA-binding protein PSF and inducing oncogene GAGE6. PLoS One 13, 1-15

24. Van Zon, A. et al. (2006) Vault mobility depends in part on microtubules and vaults can be recruited to the nuclear envelope. Exp. Cell Res. 312, 245-255

25. Horos, R. et al. (2019) The small non-coding vault RNA1-1 acts as a riboregulator of autophagy. Cell 176, 1054-1067

26. Krude, T. et al. (2009) Y RNA functions at the initiation step of mammalian chromosomal DNA replication. J. Cell Sci. 122 2836-2845

27. Hamada, M. et al. (2001) Widespread use of TATA elements in the core promoters for RNA polymerases III, II, and I in fission yeast. Mol. Cell. Biol. 21, 6870-6881

28. Mattaj, I.W. et al. (1988) Changing the RNA polymerase specificity of $U$ snRNA gene promoters. Cell 55, 435-442

29. Hernandez, N. and Lucito, R. (1988) Elements required for transcription initiation of the human $U 2$ snRNA gene coincide with elements required for snRNA $3^{\prime}$ end formation. EMBO J. 7, 3125-3134

30. Kunkel, G.R. and Pederson, T. (1988) Upstream elements required for efficient transcription of a human U6 RNA gene resemble those of $U 1$ and $U 2$ genes even though a different polymerase is used. Genes Dev. 2, 196-204

31. Lobo, S.M. and Hernandez, N. (1989) A 7 bp mutation converts a human RNA polymerase II snRNA promoter into an RNA polymerase III promoter. Cell 58, 55-67

32. Kunkel, G.R and Pederson, T. (1989) Transcription of a human U6 small nuclear RNA gene in vivo withstands deletion of intragenic sequences but not of an upstream TATATA box. Nucleic Acids Res. 17, 7371-7379

33. Murphy, S. et al. (1992) Oct-1 and Oct-2 potentiate functiona interactions of a transcription factor with the proximal sequence element of small nuclear RNA genes. Mol. Cell. Biol. $12,3247-3261$

34. Lobo, S.M. et al. (1991) The cloned RNA polymerase II transcription factor IID selects RNA polymerase III to transcribe the human U6 gene in vitro. Genes Dev. 5, 1477-1489

35. Abascal-Palacios, G et al. (2018) Structural basis of RNA polymerase III transcription initiation. Nature 553, 301-306

36. Henry, R.W. et al. (1998) SNAP19 mediates the assembly of a functional core promoter complex (SNAP ) shared by RNA polymerases II and III. Genes Dev. 12, 2664-2672

37. Vannini, A. and Cramer, P. (2012) Conservation between the RNA polymerase I, II, and III transcription initiation machineries. Mol. Cell 45, 439-446

38. Hernandez, N. (1993) TBP, a universal eukaryotic transcription factor? Genes Dev. 7, 1291-1308

39. Pugh, B.F. and Venters, B.J. (2016) Genomic organization of human transcription initiation complexes. PLoS One 11, 1-13

40. Louder, R.K. et al. (2016) Structure of promoter-bound TFIID and model of human pre-initiation complex assembly. Nature 531, 604-609

41. Kamenova, I. et al. (2014) Mutations on the DNA binding surface of TBP discriminate between yeast TATA and TATA-less gene transcription. Mol. Cell. Biol. 34, 2929-2943

42. Martinez, E. et al. (1994) TATA-binding protein-associated facor(s) in TFIID function through the initiator to direct basa 
transcription from a TATA-less class II promoter. EMBO J. 13 3115-3126

43. Haberle, V. and Stark, A. (2018) Eukaryotic core promoters and the functional basis of transcription initiation. Nat. Rev. Mol. Cell Biol. 19, 621-637

44. Knutson, B.A. and Hahn, S. (2011) Yeast Rrn7 and human TAF1B are TFIIB-related RNA polymerase I general transcription factors. Science 333, 1637-1640

45. Kostrewa, D. et al. (2009) RNA polymerase I-TFIIB structure and mechanism of transcription initiation. Nature 462 , 323-330

46. Roberts, S. et al. (1996) Cloning and functional characterization of the gene encoding the TFIIIB90 subunit of RNA polymerase III transcription factor TFIIIB. J. Biol. Chem. 271, 14903-14909

47. Emami, K.H. et al. (1997) Mechanism of synergy between TATA and initiator: synergistic binding of TFIID following a putative TFIIA-induced isomerization. Genes Dev. 11, 3007-3019

48. Kassavetis, G.A. et al. (1990) S. cerevisiae TFIIIB is the transcription initiation factor proper of RNA polymerase III, while TFIIIA and TFIIIC are assembly factors. Cell 60, 235-245

49. Kassavetis, G.A. et al. (1995) Cloning, expression, and function of TFC5, the gene encoding the B" component of the Saccharomyces cerevisiae RNA polymerase III transcription factor TFIIIB. Proc. Natl. Acad. Sci. U. S. A. 92, 9786-9790

50. Ruth, J. et al. (1996) A suppressor of mutations in the class III transcription system encodes a component of yeast TFIIIB. EMBO J. 15, 1941-1949

51. Chung, J. et al. (1987) The c-myc gene encodes superimposed RNA polymerase II and III promoters. Genes Dev. 51, 1001-1008

52. Bentley, D.L. et al. (1989) Accurate, TATA box-dependent polymerase III transcription from promoters of the c-myc gene in injected Xenopus oocytes. Genes Dev. 3, 1179-1189

53. Duttke, S.H.C. (2014) RNA polymerase III accurately initiates transcription from RNA polymerase II promoters in vitro. J. Biol. Chem. 289, 20396-20404

54. Dergai, O. et al. (2018) Mechanism of selective recruitment of RNA polymerases II and III to snRNA gene promoters. Genes Dev. 32, 1-12

55. Faresse, N.J. et al. (2012) Genomic study of RNA polymerase

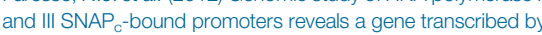
both enzymes and a broad use of common activators. PLOS Genet. 8, 1-14

56. White, R.J. (2003) Direct activation of RNA polymerase III transcription by c-Myc. Nature 421, 1698-1701

57. Hirsch, H.A. et al. (2004) Distinct mechanisms for repression of RNA polymerase III transcription by the retinoblastoma tumor suppressor protein. Mol. Cell. Biol. 24, 5989-5999

58. White, R.J. (2005) RNA polymerases I and III, growth control and cancer. Nat. Rev. Mol. Cell Biol. 6, 69-78

59. Graczyk, D. et al. (2015) Involvement of RNA polymerase III in immune responses. Mol. Cell. Biol. 35, 1848-1859

60. Cairns, C.A. and White, R.J. (1998) p53 is a general represso of RNA polymerase III transcription. EMBO J. 17. 3112-3123

61. Kiss, T. et al. (1991) Alteration of the RNA polymerase specificity of U3 snRNA genes during evolution and in vitro. Cell 65, 517-526

62. Marshallsay, C. et al. (1992) Characterization of the U3 and U6 snRNA genes from wheat: U3 snRNA genes in monocot plants are transcribed by RNA polymerase III. Plant Mol. Biol. 19, 973-983

63. Jensen, R.C. et al. (1998) The proximal sequence element (PSE) plays a major role in establishing the RNA polymerase specificity of Drosophila U-snRNA genes. Nucleic Acids Res. 26, 616-622

64. Hernandez, G. et al. (2007) Insect small nuclear RNA gene promoters evolve rapidly yet retain conserved features involved in determining promoter activity and RNA polymerase specificity. Nucleic Acids Res. 35, 21-34

65. Zamrod, Z et al. (1993) In Vitro transcription of a Drosophila U1 small nuclear RNA gene requires TATA box-binding protein and two proximal cis-acting elements with stringent spacing requirements. Mol. Cell. Biol. 13, 5918-5927

66. Waibel, F. and Filipowicz, W. (1990) U6 snRNA genes of Arabidopsis are transcribed by RNA polymerase III but contain the same two upstream promoter elements as RNA polymerase II-transcribed U-snRNA genes. Nucleic Acids Res. 18, 3451-3458

67. Connely, S. et al. (1994) Small nuclear RNA genes tran scribed by either RNA polymerase II or RNA polymerase II in monocot plants share three promoter elements and use a strategy to regulate gene expression different from that used by their dicot plant counterparts. Mol. Cell. Biol. 14, 5910-5919

68. Waibel, F. and Filipowicz, W. (1990) RNA-polymerase specificity of transcription of Arabidopsis U snRNA genes determined by promoter element spacing. Nature 346, 199-202

69. Boonanuntanasarn, S. et al. (2009) Characterization and organization of the U6 snRNA gene in zebrafish and usage of their promoters to express short hairpin RNA. Mar. Genomics 1 115-121

70. Kunkel, G.R. et al. (1996) Identification of a SPH element in the distal region of a human U6 small nuclear RNA gene promote and characterization of the SPH binding factor in HeLa cell extracts. Gene Expr. 6, 59-72

71. Korf, G.M. and Stumph, W.E. (1986) Chicken U2 and U1 RNA genes are found in very different genomic environments but have similar promoter structures. Biochemistry 25, 2041-2047

72. McNamara, K.J. et al. (1987) Transcriptional signals of a U4 small nuclear RNA gene. Nucleic Acids Res. 9239-9254

73. Stefanovic, B. and Marzluff, W.F. (1992) Characterization of two developmentally regulated sea urchin U2 small nuclear RNA promoters: a common required TATA sequence and independent proximal and distal elements. Mol. Cell. Biol. 12, $650-660$

74. Li, J.-M. et al. (1994) Transcription of the sea urchin U6 gene in vitro requires a TATA-like box, a proximal sequence element, and sea urchin USF, which binds an essential E box. Mol. Cell. Biol. 14, 2191-2200

75. Wendelburg, B.J. and Marzluff, W.F. (1992) Two promoter elements are necessary and sufficient for expression of the sea urchin UI snRNA gene. Nucleic Acids Res. 20, 3743-3751

76. Li, J.-M. et al. (1996) Common factors direct transcription through the proximal sequence elements (PSEs) of the embryonic sea urchin U1, U2, and U6 genes despite minimal sequence similarity among the PSEs. Mol. Cell. Biol. 16, 1275-1281

77. Zhao, X et al. (2001) A positioned nucleosome on the human U6 promoter allows recruitment of $\mathrm{SNAP}_{\mathrm{c}}$ by the Oct-1 POU domain. Mol. Cell 7, 539-549

78. Nikolov, D.B. et al. (1995) Crystal structure of a TFIIB-TBPTATA-element ternary complex. Nature 377, 119-128

79. Ranish, J.A. et al. (1999) Intermediates in formation and activity and a postrecruitment role for the TATA complex: holoenzyme recruitment of the RNA polymerase II preinitiation box and TFIIB. Genes Dev. 13, 49-63

80. Gouge, J. et al. (2015) Redox signaling by the RNA polymerase III TFIIB-related factor Brf2. Cell 163, 1375-1387

81. Hayashi, F. et al. (1998) Human general transcription factor TFIIB: conformational variability and interaction with VP16 activation domain. Biochemistry 37, 7941-7951

82. Zheng, L. et al. (2004) FRET evidence for a conformationa change in TFIIB upon TBP-DNA binding. Eur. J. Biochem. 271, 792-800

83. Glossop, J A et al. (2004) A conformational change in TFIBB is required for activator-mediated assembly of the preinitiation complex. Nucleic Acids Res. 32, 1829-1835

84. Mittal, V. and Hernandez, N. (1997) Role for the amino-termina region of human TBP in U6 snRNA transcription. Mol. Cell. Biol. $275,1136-1141$

85. Gouge, J. et al. (2017) Molecular mechanisms of Bdp1 in TFIIIB assembly and RNA polymerase III transcription initiation. Nat. Commun. 8, 1-10

86. McNamara-Schroeder, K.J. et al. (2001) The Drosophila U1 and U6 gene proximal sequence elements act as importan determinants of the RNA polymerase specificity of small nuclear RNA gene promoters in vitro and in vivo. J. Biol. Chem 276, 31786-31792

87. Lai, H.T. et al. (2005) The PSEA promoter element of the Drosophila U1 snRNA gene is sufficient to bring DmSNAPc into 
contact with 20 base pairs of downstream DNA. Nucleic Acids Res. 33, 6579-6586

88. Barakat, N.H. and Stumph, W.E. (2008) TBP recruitment to the U1 snRNA gene promoter is disrupted by substituting a U6 proximal sequence element A (PSEA) for the U1 PSEA. FEBS Lett. 582, 2413-2416

89. Kang, Y.S. et al. (2014) The Myb domain of the largest subunit of SNAPc adopts different architectural configurations on U1 and U6 snRNA gene promoter sequences. Nucleic Acids Res. 42, 12440-12454

90. Kim, M.K. et al. (2010) Identification of SNAPc subunit domains that interact with specific nucleotide positions in the U1 and U6 gene promoters. Mol. Cell. Biol. 30, 2411-2423

91. Li, C. et al. (2004) Architectural arrangement of cloned proximal sequence element-binding protein subunits on Drosophila $U 1$ and U6 snRNA gene promoters. Mol. Cell. Biol. 24, 1897-1906

92. Verma, N. et al. (2018) Bdp1 interacts with SNAPc bound to a U6, but not U1, snRNA gene promoter element to establish a stable protein-DNA complex. FEBS Lett. 592, 2489-2498

93. Gao, Z. et al. (2018) RNA polymerase II activity of type 3 Pol III promoters. Mol. Ther. Nucleic Acids 12, 135-145

94. Gao, Z. et al. (2019) A single H1 promoter can drive both guide RNA and endonuclease expression in the CRISPR-Cas9 sys tem. Mol. Ther. Nucleic Acid 14, 32-40

95. Bartlett, J.S. et al. (1996) Efficient expression of protein coding genes from the murine U1 small nuclear RNA promoters. Proc. Natl. Acad. Sci. U. S. A. 93, 8852-8857

96. Patel, S.B. and Bellini, M. (2008) The assembly of a spliceosomal small nuclear ribonucleoprotein particle. Nucleic Acids Res. 36, 6482-6493

97. Jeronimo, C. and Robert, F. (2017) The mediator complex: at the nexus of RNA polymerase II transcription. Trends Cell Biol. 27, 765-783
98. Zaborowska, J. et al. (2012) A novel TBP-TAF complex on RNA polymerase II-transcribed snRNA genes. Transcription 3, 92-104

99. Kuhlman, T.C. et al. (1999) The general transcription factors $\|\mathrm{A},\| \mathrm{B}, \| \mathrm{F}$, and IIE are required for RNA polymerase II transcription from the human U1 small nuclear RNA promoter. Mol. Cell. Biol. 19, 2130-2141

100. Kang, J.J. et al. (2016) TFIIIB subunit locations on U6 gene promoter DNA mapped by site-specific protein-DNA photo-crosslinking. FEBS Lett. 590, 1488-1497

101. Burton, S.P. and Burton, Z.F. (2014) The $\sigma$ enigma: bacterial $\sigma$ factors, archaeal TFB and eukaryotic TFIIB are homologs. Transcription 5, 1-8

102. Sainsbury, S. et al. (2013) Structure and function of the initially transcribing RNA polymerase II-TFIIB complex. Nature 493, 437-440

103. Hung, K.H. and Stumph, W.E. (2011) Regulation of snRNA gene expression by the Drosophila melanogaster small nuclea RNA activating protein complex (DmSNAPc). Crit. Rev. Biochem. Mol. Biol. 46, 11-26

104. Ma, B. and Hernandez, N. (2002) Redundant cooperative interactions for assembly of a human U6 transcription initiation complex. Mol. Cell. Biol. 22, 8067-8078

105. Liang, X. et al. (2003) Trans and cis splicing in trypanosomatids: mechanism, factors, and regulation. Eukaryot. Cell 2 830-840

106. Palenchar, J.B. and Bellofatto, V. (2006) Gene transcription in trypanosomes. Mol. Biochem. Parasitol. 146, 135-141

107. Das, A. et al. (2008) RNA polymerase transcription machinery in trypanosomes. Eukaryot. Cell 7, 429-434

108. Nakaar, V. et al. (1997) Structure of the Trypanosoma brucei U6 snRNA gene promoter. Mol. Biochem. Parasitol. 88 , $13-23$ 